\title{
Modelagem Matemática dos Processos de Transferência de Calor e Massa na secagem intermitente de Grãos de Soja
}

\author{
Vitor José Petry \\ UFFS - Campus Chapecó \\ 89812-000, Chapecó, SC \\ E-mail: vpetry@zipmail.com.br. \\ Pedro Elton Weber \\ UTFPR - Campus Medianeira \\ 85884-000, Medianeira, PR \\ E-mail: pedroelton@utfpr.edu.br.
}

\begin{abstract}
Resumo: O processo de secagem de grãos envolve os fenômenos de transferência de calor e de massa. Neste trabalho utilizamos um modelo de secagem cujas equações descrevem o equilíbrio de energia e massa para o ar e os grãos com base nas equações de Navier-Stokes. O coeficiente de transferência de massa por convecção entre o grão e o ar foi obtido a partir de dados experimentais para a secagem de grãos de soja. As equações do modelo são resolvidas numericamente por um esquema em diferenças finitas. As comparações entre os valores calculados pelo modelo numérico com dados experimentais para a secagem intermitente são feitas para a temperatura do ar de secagem na faixa de $50-70^{\circ} \mathrm{C}$ e para a velocidade do ar na faixa de $1,25 \mathrm{~ms}^{-1}-2,5 \mathrm{~ms}^{-1}$.
\end{abstract}

Palavras-chave: Transferência de calor e massa, secagem intermitente, solução numérica

\section{Introdução}

Em diversas aplicações práticas nos deparamos com problemas de transferência de calor e massa em meios granulares, dentre os quais destacamos o da secagem de grãos de soja. Para se ter maior segurança na secagem, garantindo a conservação da qualidade dos grãos e para evitar desperdícios, torna-se necessário o conhecimento de modelos matemáticos capazes de prever a evolução da temperatura e da umidade, tanto do ar que envolve o meio granular, como dos próprios grãos.

Existem no mercado inúmeros tipos de secadores, dentre os quais, os de leito fixo, no interior do qual uma grande massa de grãos úmidos é depositada para iniciar a secagem. A secagem artifcial dos grãos ocorre devido a um fluxo de ar que é forçado através de um sistema de ventilação a passar pelo meio granular, absorvendo a umidade contida na superfície do grão e provocando um gradiente de umidade no interior do mesmo, além do gradiente de energia, quando o fluxo é de ar quente.

Quando a distribuição de água no interior do grão não é uniforme, formam-se gradientes de concentração, fazendo com que a água se desloque dos pontos de maior concentração para os de menor concentração. Nos processos de secagem com ar aquecido, o calor é transferido para o grão e provoca a mudança de fase da água, além do aquecimento de toda a massa do grão. Formamse, assim, gradientes de pressão de vapor que também são responsáveis pelo deslocamento de água no interior dos grãos. Assim, quando a pressão de vapor parcial na superfície do grão é maior que a pressão parcial do vapor no ar, ocorre a transferência de vapor de água do grão para o ar, o que caracteriza a secagem. Quando as pressões parciais de vapor no ar e na superfície do 
grão são iguais, ocorre o equilíbrio e o teor de umidade do grão, neste caso, é chamado de teor de umidade de equilíbrio [6].

Assim, secagem de produtos agrícolas pode ser definida como um processo simultâneo de transferência de calor e massa entre o produto e o ar de secagem. De fato, quando ocorre o fluxo de ar quente por entre a massa de grãos contidos no interior da câmara de secagem, há a transferência de energia do ar para os grãos. Essa energia é rapidamente distribuída para o interior do grão, aquecendo toda a matéria e vaporizando parte da água contida no grão, aumentando a pressão parcial de vapor no interior deste e provocando, conseqüentemente, um gradiente de pressão entre o grão e o ar.

No início do processo de secagem a quantidade de calor e massa transferida é mais acentuada, diminuindo ao longo do tempo. A transferência de calor diminui à medida que a temperatura dos grãos se aproxima da temperatura do ar de secagem na entrada da câmara, tornando o gradiente de temperatura pequeno. Já a diminuição do teor de umidade dos grãos ao longo do tempo, faz com que o gradiente de umidade e de pressão de vapor entre os grãos e o ar também diminua, de forma que, quando o teor de umidade se aproxima da umidade de equilíbrio, a transferência de massa se torna praticamente desprezível.

O processo de secagem intermitente consiste em submeter os grãos a um fluxo de ar aquecido por um determinado período de tempo, seguido de outro período de tempo de secagem com ar não aquecido (temperatura ambiente), que pode ter sua passagem forçada por entre a massa de grãos por um sistema de ventilação, ou mesmo pela conveção natural, uma vez que ao final do período de secagem com ar quente, os grãos encontram-se a uma temperatura superior à temperatura do ar ambiente que está sobre o secador. A vantagem desse tipo de secagem, está na redução do consumo de energia, pois durante o período em que cessa a passagem do ar quente ocorre a redistribuição da umidade no interior dos grãos, reduzindo desta forma os gradientes de umidade e de calor. Durante este processo, continua o transporte da água até a superfície do grão, sem a necessidade de desprendimento de mais energia, de forma que quando o fluxo de ar quente é retomado, existe uma quantidade significativa de água na superfície para ser removida.

Diversos modelos e simulações tem sido feitas na tentativa de descrever os processos de secagem por diferentes autores, dentre os quais destacamos os trabalhos de Courtois et al. [3], Khatchatourian et al. [7], Liu et al. [8, 9, 10], Mhimid et al. [11], Borges [2], Srivastava and John [14], Aguerre and Suarez [1], Resio et al. [13], Gastón et al. [4], dentre outros.

Petry et al. [12] desenvolveram um modelo matemático para descrever os processos de transferência de calor e massa em um meio granular baseado nas equações de Navier-Stokes. Os termos fonte foram obtidos a partir do balanço de massa e de energia para o ar e para os grãos. Para o termo fonte da equação de transporte de massa considerou-se um coeficiente de difusão de massa entre o grão e o ar, obtendo-se uma expressão para o cálculo desse coeficiente de difusão a partir de dados experimentais.

O presente trabalho foi desenvolvido com o objetivo de observar e analisar o funcionamento do modelo no processo de secagem intermitente de grãos de soja. Dessa forma é possível verificar se os coeficientes ajustados para a secagem com temperaturas elevadas mantém o comportamento adequado do modelo quando a secagem é realizada de forma intermitente.

\section{Equações governantes e procedimentos de solução}

A câmara de secagem em leito fixo considerada nos experimentos e nas simulações numéricas é constituída por um prisma reto de base retangular. Os grãos depositados em seu interior, são considerados de medidas uniformes, em forma de esferas de $8 \mathrm{~mm}$ diâmetro.

O modelo matemático consiste de um conjunto de equações diferenciais parciais que descrevem a distribuição de temperatura e de umidade para o ar e os grãos no interior da câmara. Com a finalidade de simplificar o modelo, algumas hipótese são consideradas: a porosidade do meio granular é uniforme, não há equilíbrio térmico entre os grãos e do ar no interior da câmara, cada esfera tem temperatura uniforme, a transferência de calor do ar para os grãos ocorre por 
condução, de acordo com a lei de Fourier, a transferência de massa de água (em forma de vapor) dos grãos para o ar ocorre por difusão, obedecendo a lei de Fick, a transferência de calor no ar ocorre pelos processos de condução e advecção, a transferência de massa no ar ocorre por difusão e advecção.

O conjunto de equações governantes do modelo, na forma adimensional é dado pela equação de conservação de massa para o ar (1), equação de conservação de massa para os grãos (2), equação da energia para o ar (3) e equação de energia para os grãos (4), conforme segue:

$$
\begin{aligned}
& \frac{\partial Y^{*}}{\partial t^{*}}=-\overrightarrow{u^{*}} \cdot \vec{\nabla} Y^{*}+\frac{D^{*}}{\operatorname{ReSc}} \nabla^{2} Y^{*} \\
& +\frac{a^{*}(1-\phi)}{\phi \rho_{g}^{*} Y_{0} \operatorname{ReSc}} D_{s}^{*}\left(\rho_{s}^{*} X_{0} X^{*}-\rho_{g}^{*} Y_{0} Y^{*}\right) \\
& \frac{\partial X^{*}}{\partial t^{*}}=-\frac{a^{*}}{\rho_{s}^{*} X_{0} \operatorname{ReSc}} D_{s}^{*}\left(\rho_{s}^{*} X_{0} X^{*}-\rho_{g}^{*} Y_{0} Y^{*}\right) \\
& \frac{\partial T_{g}^{*}}{\partial t^{*}}=-\overrightarrow{u^{*}} \cdot \vec{\nabla} T_{g}^{*}+\frac{\alpha^{*}}{\operatorname{RePr}} \nabla^{2} T_{g}^{*} \\
& +\frac{a^{*}(1-\phi)}{\phi \rho_{g}^{*} C_{p_{g}}^{*} \operatorname{RePr}} K_{s}^{*}\left(T_{s}^{*}-T_{g}^{*}\right) \\
& \frac{\partial T_{s}^{*}}{\partial t^{*}}=-\frac{E c}{R e S c} \frac{a^{*} L_{v}^{*} D_{s}^{*}\left(\rho_{s}^{*} X_{0} X^{*}-\rho_{g}^{*} Y_{0} Y^{*}\right)}{\rho_{s}^{*}\left(X_{0} X^{*} C_{p_{w}}^{*}+C p_{s}{ }^{*}\right)} \\
& -\frac{1}{\operatorname{RePr}} \frac{a^{*} K_{s}^{*}\left(T_{s}^{*}-T_{g}^{*}\right)}{\rho_{s}^{*}\left(X_{0} X^{*} C_{p_{w}}^{*}+C p_{s}^{*}\right)}
\end{aligned}
$$

onde $Y$ representa a umidade do ar, $X$ o teor de umidade dos grãos, $\vec{u}$ o vetor velocidade, $D$ o coeficiente de difusão de massa de vapor no ar, $a$ a razão entre a área e o volume de um grão, $\phi$ a porosidade da massa de grãos na câmera de secagem, $\rho$ a massa específica, $T$ a temperatura, $\alpha$ a condutividade térmica, $C_{p}$ o calor específico à pressão constante e $K$ a condutividade térmica. Os subscritos $s, l, g, v$ e 0 representam o sólido, o líquido, o gás, o vapor e o valor inicial, respectivamente. $\mathrm{O}$ símbolo ${ }^{*}$ indica que a variável está na forma adimensional. $R e=\frac{U_{0} L_{c}}{\nu}$ corresponde ao número de Reynolds, $S c=\frac{\nu}{D_{0}}$ ao número de Schmid, $\operatorname{Pr}=\frac{\nu}{\alpha_{0}}$ ao número de Prandtl e $E c=\frac{U_{0}^{2}}{C_{p_{0}}\left(T_{a r}-T_{a m b}\right)}$ ao número de Eckert.

Para simplificar o modelo, os valores dos coeficientes de difusão $\left(D\right.$ e $\left.D_{s}\right)$ e do coeficiente de difusividade térmica $K_{s}$ forma considerados constantes na obtenção das equações, porém seus valores são atualizados a cada iteração do método numérico, considerando $D \propto T_{g}^{1.5}$ [5]. Para a obtenção do coeficiente de difusão de massa de água que deixa o grão e entra no ar, usou-se a expressão:

$$
D_{s}=A \sqrt{|\vec{u}|} D\left(X-X_{e}\right)^{n}
$$

onde $A$ e $n$ foram definidos a partir de dados experimentais e $X_{e}$ é a umidade de equilíbrio. Mais detalhes sobre a obtenção das equações (1 - 5) podem ser obtidas em [12].

Embora o modelo possa ser usado para descrever problemas em mais de uma direção, as simulações realizadas e a comparação com os dados experimentais foram desenvolvidas considerando o problema unidimensional. Na solução das equações do modelo foram consideradas as condições iniciais e de contorno, conforme segue:

$$
\begin{array}{ll}
T_{s}^{*}(x, 0)=0, & x \in[0,1] \\
T_{g}^{*}(x, 0)=0, & x \in(0,1]
\end{array}
$$




$$
\begin{gathered}
X^{*}(x, 0)=1, \quad x \in[0,1] \\
Y^{*}(x, 0)=1, \quad x \in[0,1] \\
T_{g}^{*}(0, t)=1 \quad e \quad \frac{\partial T_{g}^{*}}{\partial x^{*}}(1, t)=0, \quad t>0 \\
Y^{*}(0, t)=1 \quad e \quad \frac{\partial Y^{*}}{\partial x^{*}}(1, t)=0, \quad t>0
\end{gathered}
$$

Para fins de comparação com os dados experimentais, nas simulações apresentadas neste trabalho considerou-se a velocidade do ar de secagem constante.

A descretização das equações do modelo é feita utilizando o método de diferenças finitas e o método numérico para resolver o sistema linear foi o método de Gauss-Seidel.

$$
\begin{gathered}
\frac{\partial \Psi}{\partial t} \approx \frac{\Psi_{i}^{n+1}-\Psi_{i}^{n}}{\Delta t} \\
\frac{\partial \Psi}{\partial x} \approx \frac{\Psi_{i+1}^{n}-\Psi_{i-1}^{n}}{2 \Delta x} \\
\frac{\partial^{2} \Psi}{\partial x^{2}} \approx \frac{\Psi_{i+1}^{n}-2 \Psi_{i}^{n}+\Psi_{i-1}^{n}}{(\Delta x)^{2}}
\end{gathered}
$$

\section{Comparação entre dados experimentais e valores numéricos para secagem intermitente}

A secagem intermitente consiste em submeter a massa de grãos a um processo de secagem com ar quente por um determinado período de tempo, seguido por outro período em que os grãos são resfriados. Em situações reais, esse processo pode ser repetido por diversas vezes, porém nas simulações aqui apresentadas, considerou-se apenas a situação acima descrita. Em alguns casos, durante o período de resfriamento ocorre a aeração com o sistema de ventilação, desligando-se apenas o processo de aquecimento do ar. Detalhes sobre a obtenção dos dados experimentais são apresentados em Weber et. al. [15] e são usados neste trabalho para fins de comparações com os valores obtidos pelo modelo numérico.

Apresentamos na sequência comparações entre dados experimentais e valores numéricos para simulações com diferentes valores da temperatura do ar de secagem na entrada da câmara, da velocidade do ar de secagem, da umidade inicial dos grãos a serem secados e da temperatura ambiente. Nestas simulações são obtidas curvas que descrevem a temperatura do ar, a umidade dos grãos e a temperatura dos grãos no interior da câmara. Os resultados são apresentados para quatro posições distintas da câmara de secagem $\left(x^{*}=1 / 8, x^{*}=3 / 8, x^{*}=5 / 8\right.$ e $\left.x^{*}=7 / 8\right)$. Os valores da temperatura e da umidade estão na forma adimensional.

A primeira simulação foi realizada com temperatura ambiente $T_{a m b}=23^{\circ} \mathrm{C}$, temperatura do ar de secagem na entrada da câmara $T_{a r}=70^{\circ} \mathrm{C}$, umidade inicial dos grãos $X_{0}=0,214$, velocidade do ar na entrada da câmara $U_{0}=1,25 \mathrm{~ms}^{-1}$ e umidade relativa do ar ambiente $U R=48,7 \%$.

Nas simulações apresentados na figura 1 considera-se três horas de secagem com ar aquecido, seguido de cinquenta minutos de aeração com ar a temperatura ambiente.

Os comparativos entre valores numéricos e dados experimentais para a distribuição do teor de umidade dos grãos de soja obtidos na primeira simulação são mostrados na figura 2. Já as curvas de distribuição da temperatura dos grãos de soja para as mesmas condições da primeira simulação, porém com 45 minutos de secagem com ar quente, seguidos de 50 minutos de aeração com temperatura igual à temperatura ambiente, são apresentados na Figura 3.

Observa-se boa concordância entre os valores obtidos numericamente com os dados experimentais, principalmente para as curvas que mostram o teor de umidade dos grãos (figura 2). Já 


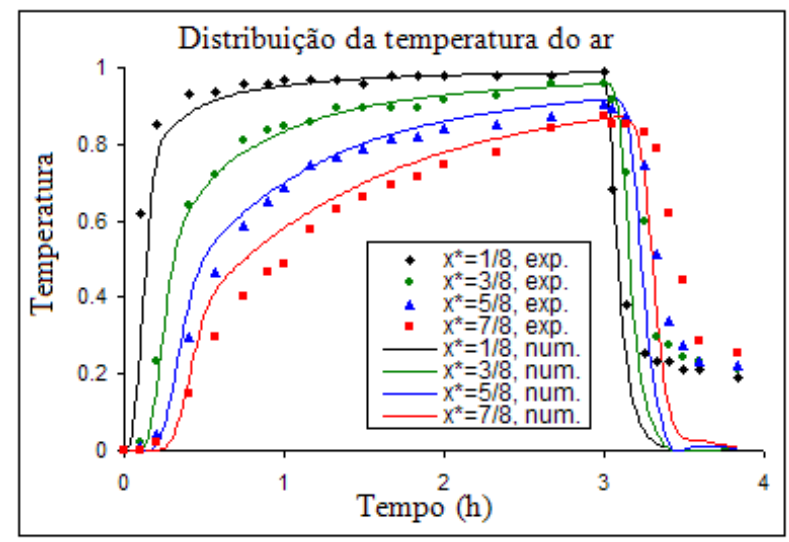

Figura 1: Distribuição da temperatura do ar para $T_{a m b}=23^{\circ} C, T_{a r}=70^{\circ} C, X_{0}=0,214$ e $U_{0}=$ $1,25 \mathrm{~ms}^{-1}$

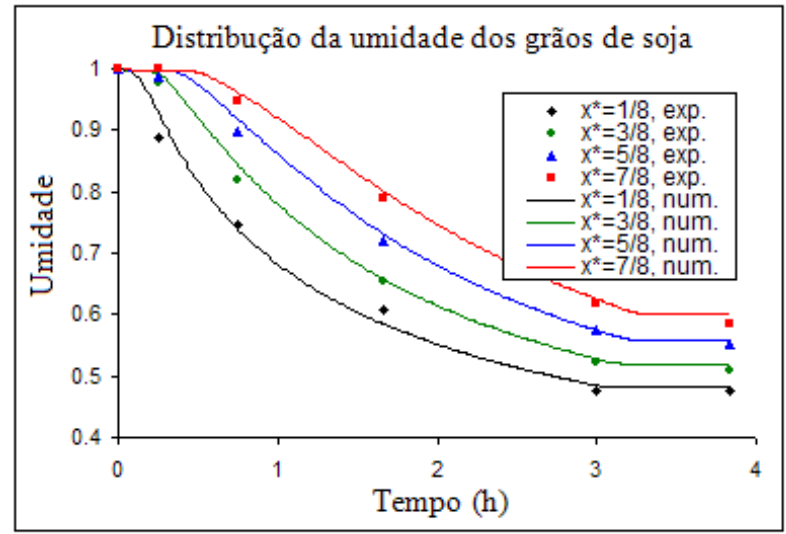

Figura 2: Distribuição da umidade dos grãos de soja para $T_{a m b}=23^{\circ} C, T_{a r}=70^{\circ} C, X_{0}=0,214 \mathrm{e}$ $U_{0}=1,25 \mathrm{~ms}^{-1}$

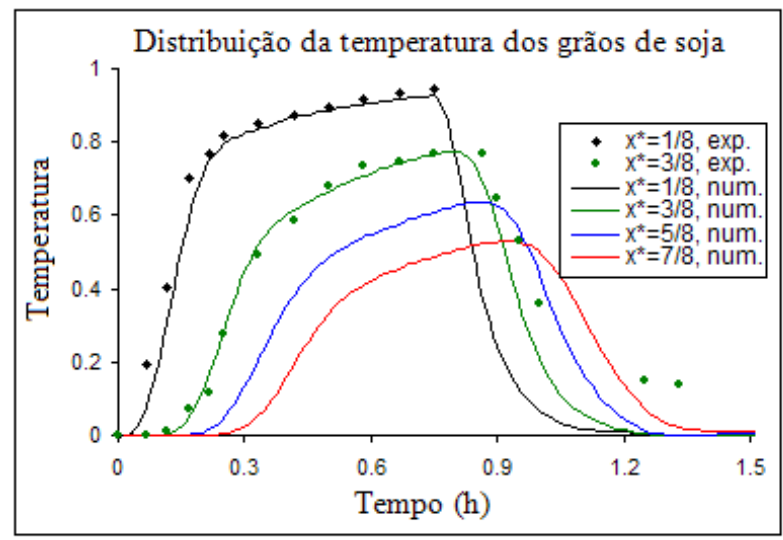

Figura 3: Distribuição da temperatura dos grãos de soja para $T_{a m b}=23^{\circ} \mathrm{C}, T_{a r}=70^{\circ} \mathrm{C}, X_{0}=0,214 \mathrm{e}$ $U_{0}=1,25 \mathrm{~ms}^{-1}$

no caso das curvas de temperatura, observa-se um rápido desvio entre os valores numéricos e experimentais na parte final da câmara. Como possíveis causas para esses desvios, apontamos 
as perdas de calor na saída da câmara, não consideradas no modelo, e a eventual interferência do meio granular no processo de convecção de forma que no modelo, onde essas situações não são consideradas, a temperatura na parte final da câmara de secagem decresce mais rapidadente do que na situação real (experimento).

Outra simulação foi realizada para temperatura ambiente $T_{a m b}=13^{\circ} \mathrm{C}$, temperatura do ar de secagem na entrada da câmara $T_{a r}=50^{\circ} \mathrm{C}$, teor de umidade inicial do produto $X_{0}=0,227$, velocidade do ar de secagem na entrada da câmara $U_{0}=2,5 \mathrm{~ms}^{-1}$ e umidade relativa do ar ambiente $U R=70 \%$. As curvas da temperatura do ar dessa simulação são mostradas na figura 4 , enquanto na figura 5 compara-se os valores do teor de umidade do produto ao longo do tempo, referentes à mesma simulação.

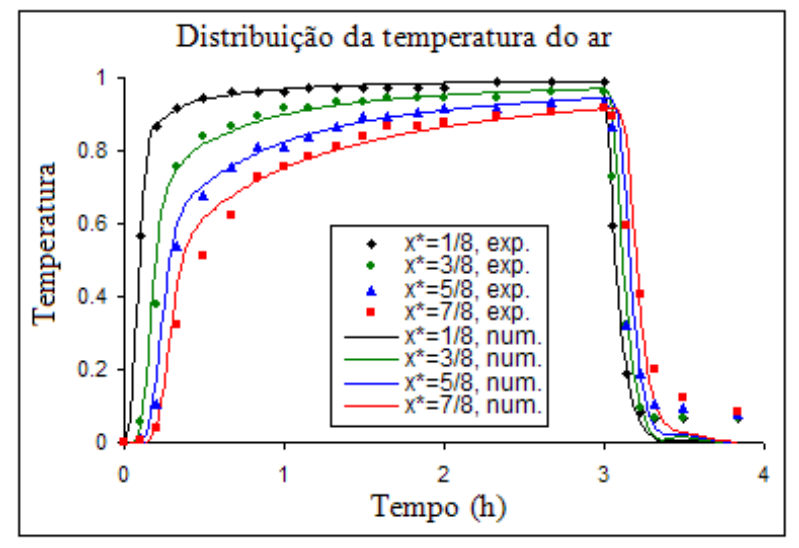

Figura 4: Distribuição da temperatura do ar para $T_{a m b}=13^{\circ} C, T_{a r}=50^{\circ} C, X_{0}=0,227$ e $U_{0}=$ $2,5 \mathrm{~ms}^{-1}$

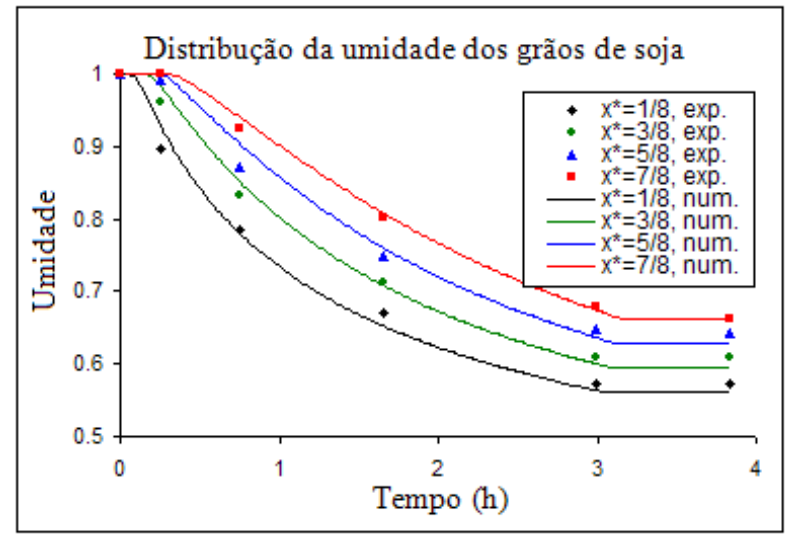

Figura 5: Distribuição da umidade dos grãos de soja para $T_{a m b}=13^{\circ} C, T_{a r}=50^{\circ} C, X_{0}=0,227 \mathrm{e}$ $U_{0}=2,5 \mathrm{~ms}^{-1}$

\section{Conclusões}

Neste trabalho usamos um modelo matemático que descreve os processos de calor e massa em um meio granular para simular o processo de secagem intermitente de grãos de soja. As simulações numéricas foram realizadas usando um esquema em diferenças finitas e os resultados obtidos indicam uma boa concordância entre dados experimentais e valores numéricos obtidos nas simulações para uma faixa de variação da temperatura do ar de secagem $T_{a r}=50^{\circ} \mathrm{C}-70^{\circ} \mathrm{C}$ 
e velocidade do ar na faixa de $1.25 \mathrm{~ms}^{-1}-2.5 \mathrm{~ms}^{-1}$. Esses resultados contribuem para uma melhor compreensão do processo de secagem que ocorre no interior da câmara.

\section{Referências}

[1] R.J. Aguerre and C. Suarez, Diffusion of bound water in starchy materials: application to drying, Journal of Food Engineering 64 (2004) 389-395.

[2] P.A.P. Borges, Modelling of the involved processes in the grains drying and storage systems, PhD thesis, PROMEC/UFRGS, Porto Alegre, BR, (2002).

[3] F. Courtois, A. Lebert, J.C. Lasseran and J.J. Bimbenet, Simulation of industrial dryers: solving numerical and computer problems, Drying Technology 9(4) (1991) 927-945.

[4] A.L. Gastón, R.M. Abalone, S.A. Giner and D.M. Bruce, Effect of modelling assumptions of the effective water diffusivity in wheat, Biosystems Engineering 88(2) (2004), 175-185.

[5] F.P. Incropera, Fundamentals of heat and mass transfer, Wiley, New York, (1990).

[6] J. C. Lasseran, Practical experiences on aeration-drying. Revista Brasileira de Armazenamento. 6(1) (1981) 13-23.

[7] O. Khatchatourian, P.A.P Borges and V.J. Petry, Mathematical model and numerical methods for the simulation of dynamics of drying of soy grains in camera of fixed bed, Proceedings of the V LACAFLUM, Caracas, (2001) 1-6.

[8] Q. Liu, M.D. Montross and F.W. Bakker-Arquema, Stochastic modeling of grain drying. Part 1: experimental investigation, Journal Agricultural Engineering Research 66 (1997) 267-274.

[9] Q. Liu and F.W. Bakker-Arquema, Stochastic modeling of grain drying. Part 2: model development, Journal Agricultural Engineering Research 66 (1997) 275-280.

[10] Q. Liu and F.W. Bakker-Arquema, Stochastic modeling of grain drying. Part 3: analysis of crossflow drying, Journal Agricultural Engineering Research 66 (1997) 281-286.

[11] A. Mhimid, S. Ben Nasrallah and J.P. Forh, Heat and mass transfer during drying of granular products - simulations with convective and conductive boundary conditions, International Journal of Heat and Mass Transfer 43 (2000) 2779-2791.

[12] V.J. Petry, A.L. De Bortoli and O. Khatchatourian, Development of a mathematical model for heat and mass transfer inside a granular medium, Proceedings of the Computational Methods in Engineering and Science, Sanya, Hainan, China (2006) 649-655.

[13] A.N.C. Resio, R.J. Aguerre and C. Suarez, Drying characteristics of amaranth grain, Journal of Food Engineering 65 (2004) 197-203.

[14] V.K. Srivastava and J. John, Deep bed grain drying modelling, Energy Conversion and Management 43 (2002) 1689-1708.

[15] P. E. Weber, Khatchatourian O., Borges P. Numerical simulation of soy drying in a continuous flow dryer. In. Proceedings of the $16^{\text {th }}$ Brazilian Congress of Mechanical Engineering - COBEM 9, Uberlândia (2001) 300-307. 